\title{
African Meridian B-Field Education and Research (AMBER) Array
}

\author{
E. Yizengaw $\cdot$ M. B. Moldwin
}

Received: 2 October 2007/ Accepted: 12 June 2008/Published online: 17 January 2009

(C) The Author(s) 2009. This article is published with open access at Springerlink.com

\begin{abstract}
The AMBER array contains four magnetometers and spans across the geomagnetic equator from $\mathrm{L}$ of 1 to an $\mathrm{L}$ of 1.4. In addition to filling the largest land-based gap in global magnetometer coverage, the AMBER array will address two fundamental areas of space physics: (1) the processes governing electrodynamics of the equatorial ionosphere as a function of latitude (or L-shell), local time, longitude, magnetic activity, and season, and (2) ULF pulsation strength and its connection with equatorial electrojet strength at low/ mid-latitude regions. Satellite observations show unique equatorial ionospheric structures in the African sector, though these have not been confirmed by observation from the ground due to lack of ground-based instruments in the region. In order to have a complete global understanding of equatorial ionosphere motions, deployment of ground-based magnetometers in Africa is essential. One focus of IHY is the deployment of networks of small instruments, including the development of research infrastructure in developing nations through the United Nations Basic Space Science (UNBSS) Small Instrument Array. Therefore, AMBER magnetometer array in partnership with parallel US funded GPS receivers in Africa will allow us to understand the electrodynamics that governs equatorial ionosphere motions. While AMBER routinely observes the F region plasma drift mechanism $(\mathbf{E} \times \mathbf{B}$ drift $)$, the GPS stations will monitor the structure of plasma at low/midlatitudes in the African sectors. In addition to new scientific discoveries and advancing the space science research into Africa by establishing scientific collaborations between scientists in the developing and developed nations, the AMBER project also contributes to developing the basic science of heliophysics through cross-disciplinary studies of universal process. This includes the creation of sustainable research/training infrastructure within the developing nations (Africa).
\end{abstract}

Keywords AMBER $\cdot$ Equatorial electrojet $\cdot$ ULF pulsation $\cdot$ IHY

E. Yizengaw $(\bowtie) \cdot$ M. B. Moldwin

Institute of Geophysics and Planetary Physics, University of California, Los Angeles, CA, USA

e-mail: ekassie@igpp.ucla.edu 


\section{Introduction}

Understanding the fundamental processes governing the electrodynamics and plasma production and loss in the low/mid-latitudes region is essential for space weather forecasting. The equatorial anomaly (EA) has been well-described as arising from the electrodynamics at the equator. The anomaly is formed through the combined effects of $\mathbf{E} \times \mathbf{B}$ drift and ambipolar diffusion, where E represents the electric field, and B the geomagnetic field. During the day an eastward electric field generated in the E region of the ionosphere through dynamo action (e.g., Tarpley 1970) and/or penetrate to the ionosphere from the magnetosphere (e.g., Mannucci et al. 2005) causes $F$ region plasma to drift upward with a velocity of $\mathbf{E} \times \mathbf{B} / \mathbf{B}^{2}$. At the same time gravitational and pressure gradient forces move ionization downward along the magnetic field lines thus transporting plasma away from the magnetic equator toward higher latitudes. In this way ionization crests are formed north and south of the dip-equator. Solution of the time independent plasma continuity equation including production, loss, diffusion and $\mathbf{E} \times \mathbf{B}$ drift (e.g., Anderson 1973) yields a noontime latitudinal electron density $\left(\mathrm{N}_{\mathrm{e}}\right)$ distribution with peaks at $\pm 20^{\circ}$ geomagnetic latitude for a drift of $15 \mathrm{~m} / \mathrm{s}$. In the absence of drift the peaks form at $\pm 8^{\circ}$ latitude.

The EA regions are of importance due to their connection with the equatorial ionosphere, and their location as the latitudinal transition region from low-latitudes into the tropical and mid-latitudes. The ionosphere at the equator and low-latitudes is the site of important post-sunset ionospheric phenomena particularly during solar maximum. These include the enhanced equatorial or Appleton anomaly, bottom side spread F (BSSF), and equatorial bubbles. The latter two are often described together as equatorial spread $\mathrm{F}$ (ESF), although they have separate identities as to altitude, latitude, and continuity in LT. These irregularities are extremely dynamic and highly disruptive, causing signal deterioration to communication and navigation systems (Klobuchar et al. 1991). The large-scale irregularities extend over large ranges of latitude, longitude, and time. They also cause ESF conditions (dropouts in the signal levels in the HF spectrum) to small-scale irregularities that cause scintillation (deterioration of the quality of signals for ground to satellite communications). Scintillation causes loss of lock on a satellite, which can degrade GPS positional accuracy and reliability. Earlier studies show that strong BSSF is a necessary condition for bubble formation and is related to maximum pre-reversal $\mathbf{E} \times \mathbf{B}$ drift over a large range of solar flux and magnetic activity. The crest of the anomaly contains the highest levels of $\mathrm{N}_{\mathrm{e}}$ on earth and its intersection with the bubble gives rise to the highest levels of scintillation (both amplitude and phase on trans-ionospheric propagation frequencies (Klobuchar et al. 1991)) levels that are greatest during solar maximum.

The EA has been studied for many years; this includes its dependence of ESF, on $\mathbf{E} \times \mathbf{B}$ drift (e.g., Fejer et al. 1999) and the relation between ESF and the anomaly (e.g., Mendillo et al. 2001). Modeling of the relation of $\mathbf{E} \times \mathbf{B}$ drift to the EA has also had a long history (e.g., Balan and Bailey 1995). Even though it has become a challenging region for our navigation and communication systems, equatorial ionospheric instability studies have been conducted less in the African sector. There has been one experimental campaign conducted in the region during the years 1993-1994 in the frame work of the International EEJ Year (IEEY) with 10 magneto-telluric stations and a network of three ionosonde (e.g., Amory-Mazaudier et al. 2005 and the references therein). Almost all of the instruments were brought to Africa, in particular only in West African region, for the IEEY experiment campaign and now are not in use. Their unique observational results in the region are reviewed in detail and documented in (Amory-Mazaudier et al. 2005). 
Systematic and extensive observations are required for complete global understanding of the fundamental processes governing electrodynamics of the equatorial ionosphere. However, the uneven distribution of ground-based instruments hinders our ability to obtain a global understanding of the equatorial ionosphere. In the African sector, for example, a continuous observation of the EA irregularity is not possible due to lack of ground-based instruments, causing gaps of understanding of the space weather phenomenon in the region. Therefore, to have a complete and global understanding of the electrodynamics of the equatorial ionosphere in a cost effective way, deployment of small instruments, like ground-based magnetometers and GPS receivers, is essential.

The routine measurement of ULF waves with networks of ground-based magnetometers can also be used to track the transfer of energy from the solar wind to the inner-magnetosphere at one local time and also provide a useful proxy for determining the state of the magnetosphere at a given time along a given set of field lines (e.g., Berube et al. 2003). However, ULF pulsations at low L-shell and their relation to equatorial electrojet (EEJ) and EA phenomena are less clear. Ground-based observations show that ULF pulsations increase in amplitude toward the geomagnetic equator, believed to be an EEJ phenomenon (e.g., Pathan et al. 1999). To understand ULF pulsations at low L-shell and their relationship to EEJ and EA, simultaneous measurements of ULF wave power, EEJ, and EA at low/mid-latitudes is crucial. Therefore, the NASA-IHY funded AMBER magnetometer network will play a vital role in providing the necessary monitoring in the EuropeanAfrican sector, a region which has been relatively devoid of ground-based instruments at low-latitudes in the African continent. At the same time similar observations can be performed in the American sector using CARISMA (formerly CANOPUS) (Rostoker et al. 1995), MEASURE (Berube et al. 2003), and SAMBA (Zesta et al. 2004) magnetometer arrays so that we will be able to examine the longitudinal dependence of these features.

The processes of energetic particle acceleration in the inner-magnetosphere remains in general poorly understood. Recent theoretical and observational developments have highlighted the possibility that ULF waves accelerate electrons to $\mathrm{MeV}$ energies in the outer radiation belt (e.g., Mann et al. 2004 and the reference therein). Evidence shows that ULF waves correlated with $\mathrm{MeV}$ electron flux propagating radially inwards from $L=6.6$, to $L=5.5$, to $L=4.5$ (e.g., Mann et al. 2004). However, its acceleration mechanism at low L-shell remains unclear (see Loto'aniu et al. 2006). Therefore, to understand the radiation belt dynamics in the inner belt, ULF wave measurements at multiple meridians along the electron drift-trajectory is crucial. This is especially true if the relative importance of competing ULF (e.g., Loto'aniu et al. 2006) and VLF (e.g., Horne et al. 2005) wave acceleration is to be resolved. AMBER will provide important monitoring opportunity in the European-African sector by extending the European magnetometer arrays to low and dip-equator latitudes. This coverage is of particular importance to studies of enhancements of $\mathrm{MeV}$ energy electrons in the slot and the inner zone (Baker et al. 2004); for example Loto'aniu et al. (2006) suggest that ULF waves at low L-shell have been responsible for the observed slot and inner belt electron enhancements during the onset of the "Halloween" 2003 superstorm period on 29th October, 2003.

\section{AMBER Instrumentation}

The ground-based fluxgate magnetometers of the NASA-IHY funded AMBER array have their heritage in the space-based instruments that have been developed at UCLA over the last few decades. The sensor is designed to be buried approximately $1 \mathrm{~m}$ into the ground 


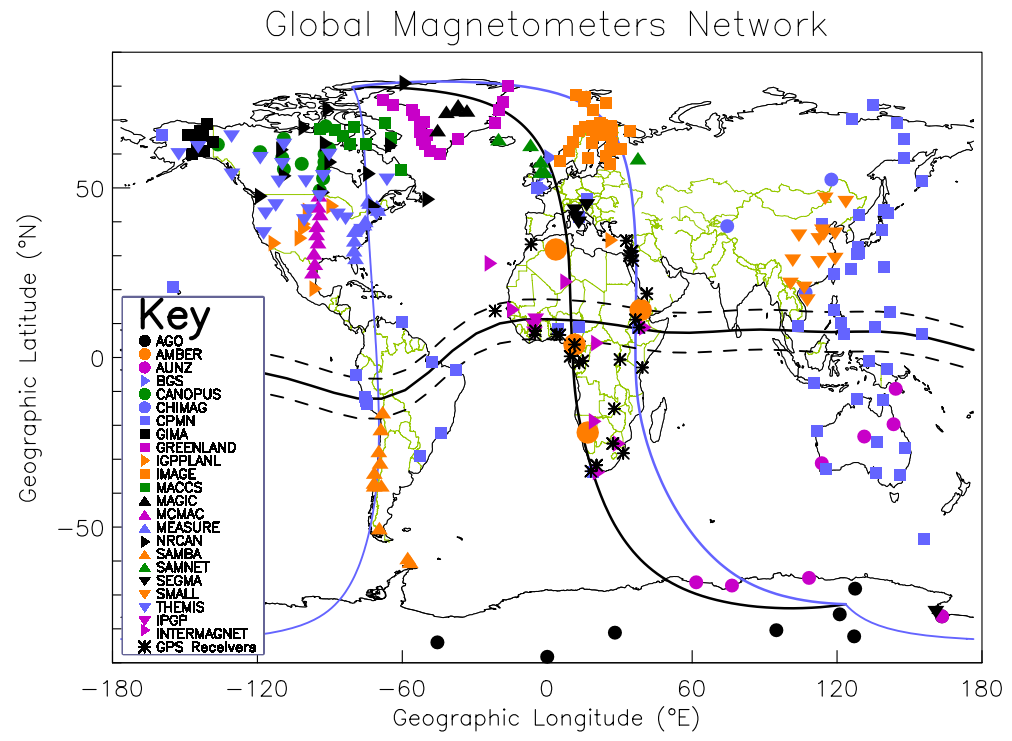

Fig. 1 Global magnetometer coverage along with proposed AMBER array (red dots)

Table 1 List of the locations of the proposed AMBER magnetometer stations

\begin{tabular}{llccrrr}
\hline Name of stations & Code & Geo. Lat & Geo. Long & Mag. Lat & Mag. Long & L-shell \\
\hline Algiers, Algeria & ALA & $36.85^{\circ} \mathrm{N}$ & $2.93^{\circ} \mathrm{E}$ & $27.98^{\circ} \mathrm{N}$ & $77.67^{\circ} \mathrm{E}$ & 1.3 \\
Adigrat, Ethiopia & ADI & $14.28^{\circ} \mathrm{N}$ & $39.46^{\circ} \mathrm{E}$ & $5.80^{\circ} \mathrm{N}$ & $111.06^{\circ} \mathrm{E}$ & 1.0 \\
Windhoek, Namibia & WIN & $22.56^{\circ} \mathrm{S}$ & $17.09^{\circ} \mathrm{E}$ & $33.15^{\circ} \mathrm{S}$ & $84.65^{\circ} \mathrm{E}$ & 1.4 \\
Yaounde, Cameroon & YAC & $3.87^{\circ} \mathrm{N}$ & $11.52^{\circ} \mathrm{E}$ & $5.30^{\circ} \mathrm{S}$ & $83.12^{\circ} \mathrm{E}$ & 1.0 \\
\hline
\end{tabular}

and is housed in an oil bath to help eliminate temperature fluctuations. The sensor is connected to the PC-based electronics card via a 30 or $60 \mathrm{~m}$ cable. The data are sampled at $0.5 \mathrm{~Hz}$ and are time tagged with the accuracy of $10^{-6} \mathrm{~s}$. This sensor has been operating in the field as part of the MEASURE and UCLA/IGPP arrays for several years. The magnetometers are fabricated and tested completely in-house.

The AMBER magnetometer array is comprised of four magnetometers, which will be stationed in Algiers in Algeria, Yaounde in Cameroon, Windhoek in Namibia, and Adigrat in Ethiopia. Figure 1 shows the global magnetometer network. AMBER array (red dots in the figure) will cover from the $L$ value of 1 to $L$ of $1.4\left(36.85^{\circ} \mathrm{N}-22.56^{\circ} \mathrm{S}\right.$ geographic). The specific location of each AMBER magnetometer is shown in Table 1. While polar-cap to equatorial coverage will exist in the American meridian with the deployment of the NSF funded McMAC array linking the expanded Canadian CARISMA (formerly CANOPUS) and the SAMBA arrays, in Europe the coverage essentially ends with SEGMA in Italy. AMBER array, which will be fully operational in 2008, will then connect the European magnetometer array to low-latitudes by filling the largest land-based gap in global magnetometer coverage in Africa, as is shown in Fig. 1.

AMBER stations in Algiers, Yaounde, and in Windhoek will be used for connecting IMAGE (Ritter et al. 2004)-SAMNET (e.g., Mann et al. 2004)-SEGMA array to low and 
dip-equator latitudes, and link up with South African Intermagnet and Antarctic magnetometers in the southern hemisphere so that we can have complete meridian observation in the region. We will combine the stations in Adigrat and in Yaounde with MAGDAS (formerly known as CPMN) (Yumoto 2006) stations in Addis Ababa, Ethiopia and in Ilorin, Nigeria, respectively, and routinely monitor the motion of the plasma at low/mid-latitudes by continuously estimating the strength of EEJ and thus $\mathbf{E} \times \mathbf{B}$ drift at two different meridians. The AMBER station in Adigrat is located at $\sim 6.0^{\circ} \mathrm{N}$ off from the equator and MAGDAS in Addis Ababa is located nearly at the equator $\left(\sim 0.13^{\circ} \mathrm{N}\right.$ magnetic $)$. Similarly, the AMBER station at Yaounde is located at $\sim 5.30^{\circ} \mathrm{S}$ off from the equator while MAGDAS in Ilorin is situated at $\sim 1.5^{\circ} \mathrm{S}$ magnetic. Data will be returned from AMBER stations using internet connectivity through African Universities and will be made immediately available on the World Wide Web and will be able to provide extensive mid- and low-latitude coverage. This data will then be directly accessible to space weather forecasters and the space science community at large. As a final back-up option (or for use during periods of long-lasting internet interruption), data collection could also be done if necessary by mailing CDs. Regardless of how the data are shipped to the database, all data will be online within oneweek (internet accessible sites) or two months (non-connected sites).

\section{Description of Techniques and Technical Background}

At mid- and low-latitudes the effects of vertical $\mathbf{E} \times \mathbf{B}$ drifts begin to strongly influence ionospheric structure (e.g., Anderson et al. 2000). The schematic diagram shown in Fig. 2a presents the configuration of B-field, E-field, and the direction of $\mathbf{E} \times \mathbf{B}$ plasma drift. The plasma is lifted at the equator and then moves down along the magnetic field line to higher latitude regions by the pressure gradient and gravity forces as shown in the figure. The magnitude and direction of $\mathbf{E} \times \mathbf{B}$ drift can be easily estimated using the ground-based magnetometer array (Anderson et al. 2000). This is possible since the horizontal

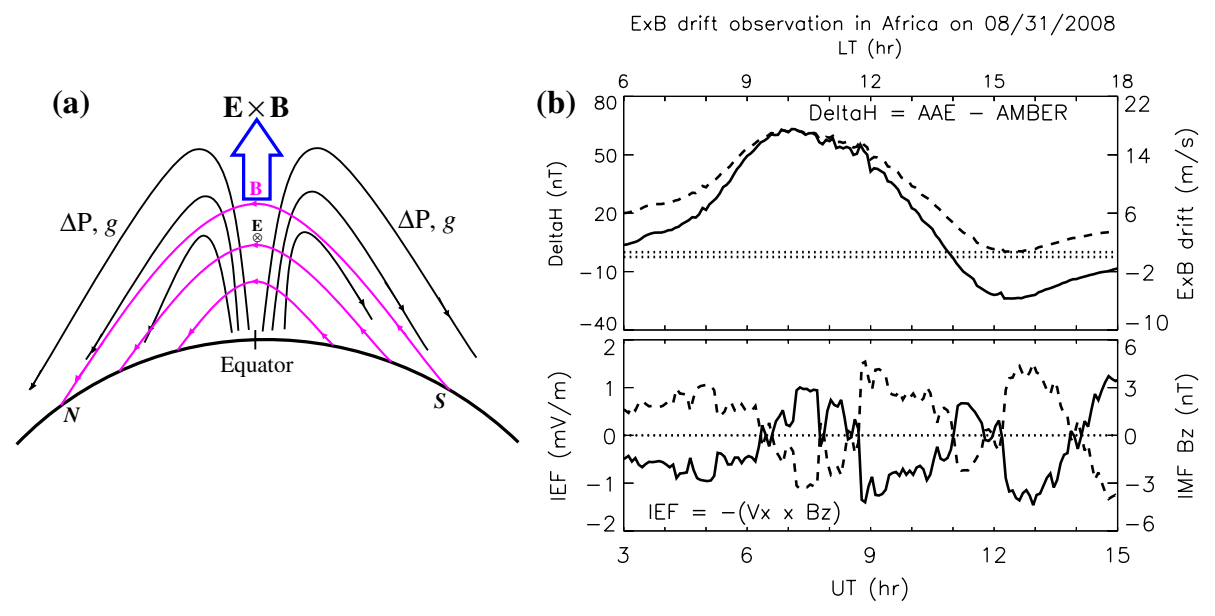

Fig. 2 a Schematic diagram showing the equatorial fountain effect (after Yizengaw et al. 2005a). b The $\Delta H$ (solid curve) obtained from the magnetometer data at equator and off the equator to estimate $\mathbf{E} \times \mathbf{B}$ drift (dashed curve), and at the bottom panel shows the magnetospheric electric field (solid curve) estimated from ACE data and IMF Bz (dashed curve) 
component of the geomagnetic field at the magnetic dip-equator reflects the change in the equatorial plasma fountain, and the difference between $H$ at the equator and at a nonequatorial location is a good indicator of the vertical $\mathbf{E} \times \mathbf{B}$ drift (Anderson et al. 2000; Yizengaw et al. 2004, 2005a). In the ionospheric dynamo, the $H$-component reflects the EEJ current (Anderson et al. 2000; Yizengaw et al. 2004, 2005a). Therefore, using the magnetometer data from MAGDAS (Yumoto 2006) and the AMBER array and by applying the technique described in (Anderson et al. 2000; Yizengaw et al. 2004, 2005a) we will continuously estimate the $\mathbf{E} \times \mathbf{B}$ drift. This can be possible by differencing $H$-component field recorded at a non-equator magnetometer $\left(H_{\text {non-equator }}\right)$ from the $H$-component value measured by a magnetometer at the magnetic equator $\left(H_{\text {equator }}\right)$. We will perform these independent observations $\left(\Delta H=H_{\text {equator }}-H_{\text {non-equator }}\right)$ at two different meridians (at $10^{\circ} \mathrm{E}$ and $40^{\circ} \mathrm{E}$ geographic) to study its longitudinal dependence as well. A pair of AMBER stations in Cameroon and MAGDAS in Nigeria (see Fig. 1) will be used to estimate $\mathbf{E} \times \mathbf{B}$ drift at $\sim 10^{\circ}$ E longitudes. Similarly, a pair of AMBER and MAGDAS stations in Ethiopia will also routinely estimate the strength of the EEJ and thus $\mathbf{E} \times \mathbf{B}$ drift at $\sim 40^{\circ} \mathrm{E}$ longitudes. Top panel in Fig. $2 \mathrm{~b}$ shows a typical example of indirectly estimated $\mathbf{E} \times \mathbf{B}$ drift (dashed curve) and parameter $(\Delta H)$ (solid curve) obtained from AMBER magnetometer data at Adigrat $\left(5.80^{\circ} \mathrm{N}, 111.06^{\circ} \mathrm{E}\right.$ geomagnetic) and Intermagnet station at Addis Ababa $\left(0.13^{\circ} \mathrm{N}, 111.10^{\circ} \mathrm{E}\right.$ geomagnetic) observed on 31 August 2008 . We also used ACE data to estimate the magnetospheric electric field (solid curve in the bottom panel) to understand its role, as prompt penetration electric field when IMF Bz (dashed curve) turns south, for the processes governing electrodynamics of the equatorial ionosphere. This can be done by comparing the magnetospheric electric field $\left(v_{x} \times B_{z}\right)$ and $\mathbf{E} \times \mathbf{B}$ drift estimated using ground-based magnetometer data as shown in Fig. 2 b.

Simultaneously, we will monitor the structure of EA using GPS TEC data. A twodimensional TEC plot will be used to reveal the latitudinal and longitudinal extent of the EA. In addition to the ground-based GPS receivers, the LEO satellites equipped with dualband frequency GPS receivers offer the opportunity for remote sensing and monitoring of the topside ionosphere and plasmasphere regions by providing the integrated $\mathrm{N}_{\mathrm{e}}$ between the LEO and GPS satellites. This can be done since GPS receivers on the ground and on board continuously receive dual frequency signals transmitted by GPS satellites that broadcast two L-band signals at frequencies $f_{1}=1.57542 \mathrm{GHz}$ and $f_{2}=1.2276 \mathrm{GHz}$. The integrated $\mathrm{N}_{\mathrm{e}}$ or TEC along the ray path between the GPS receiver and a GPS satellite can be derived from the combination of GPS pseudoranges $\left(P_{1}-P_{2}\right)$ and carrier phases $\left(L_{1}-L_{2}\right)$ (Sardón et al. 1994; Yizengaw et al. 2004). It is convenient to divide the LEO GPS data into two types: GPS below the horizon data that is recorded when LEO satellites detect GPS signals that have traversed the ionosphere below LEO satellites' orbiting height, GPS above the horizon data that is recorded when LEOs receive signals that have crossed the ionosphere above its orbiting height. The LEO GPS data is pre-processed to remove outlier values and cycle slips, which are either removed or corrected.

Tomographic reconstruction technique (e.g., Yizengaw and Moldwin 2005; Yizengaw et al. 2005a) will also be applied to the GPS TEC to image to F region height rise (see typical example in Fig. 3) in particular and the altitude extension of bubbles in the African region. We will use the topside tomography (Heise et al. 2002; Yizengaw et al. 2005b, 2006) to monitor the maximum plasma height extension at the equator. We will then do case studies of EA and $\mathbf{E} \times \mathbf{B}$ comparison by taking the advantage of ground-based magnetometer and ground- and space-based TEC. Because, while magnetometer provides the different strength stages of EEJ or $\mathbf{E} \times \mathbf{B}$, GPS data provide multiple snapshots of EA and/or bubble at different stages of their evolution. We will also examine the relation 

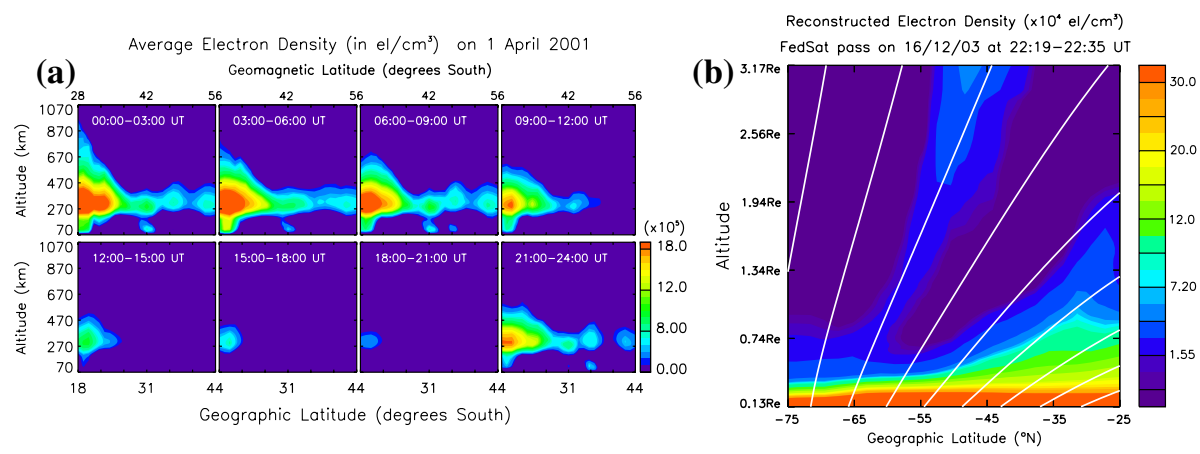

Fig. 3 a Images of ionospheric $N_{e}$ in altitude versus geographic latitude plane for 1st April 2001 (Kp > 8) (after Yizengaw et al. 2007). b Tomographically reconstructed density distribution using data recorded by GPS receiver on FedSat. The curved white lines depict the magnetic field lines obtained from Tsyganenko01 model (after Yizengaw et al. 2006)

between EEJ or $\mathbf{E} \times \mathbf{B}$ and the formation and evolution of EA, bubble, ESF, and BBSF statistically by binning the observations into local time (LT) and geomagnetic activity bins.

Figure 3a shows the ground-based tomographic reconstruction of $\mathrm{F}$ region ionosphere for 1st April 2001. It shows a region of enhanced $\mathrm{N}_{\mathrm{e}}$ extending up to an altitude of $950 \mathrm{~km}$. This feature was more pronounced during pre-noon and remained present until midnight $(L T=U T+10)$. It also expanded to the south (poleward) up to $\sim 27^{\circ} \mathrm{S}\left(\sim 38^{\circ} \mathrm{S}\right.$ geomagnetic). In the afternoon this $\mathrm{N}_{\mathrm{e}}$ enhancement decreases in magnitude and migrates back to lower latitudes, tending to disappear post midnight. These series of plots are of interest because they show evidence for the equatorial anomaly. Recently, we have accomplished one case study and tomographically imaged the flux tube structure of ionospheric ion outflows (see Fig. 3b), tracking its structure up to the 3.17Re $(20,200 \mathrm{~km}$ or GPS orbiting height) altitude (Yizengaw et al. 2006). Figure 3b shows a typical tomographically reconstructed $\mathrm{N}_{\mathrm{e}}$ distribution in a two-dimension (altitude versus latitude) space region derived from one of LEO satellite (FedSat) north-to-south pass, covering 5.0-75.0 ${ }^{\circ} \mathrm{S}$ geographic at about $170^{\circ} \mathrm{E}$ longitude and between 22:10 UT and 22:35 UT on 16 December 2003. The altitude range shown extends from $800 \mathrm{~km}(0.13 \mathrm{Re})$ to $20,200 \mathrm{~km}$ (3.17Re). The white lines depict magnetic field lines generated using the Tsyganenko-01 model (Tsyganenko 2002). Note the presence of a plume of plasma at the cusp latitude.

The ULF pulsation strength at low L-shell and its relation with EEJ and EA is not well understood. Ground-based observation shows that ULF pulsations increase in amplitude toward the geomagnetic equator, believed to be an EEJ phenomenon (e.g., Pathan et al. 1999). To understand ULF pulsation at low L-shell and its relation with EEJ and EA, simultaneous measurements of ULF pulsation, EEJ, and EA at low/mid-latitude region is crucial. We will use the Europe-Africa magnetometer array link to continuously monitor the ULF power as function of latitude. We will combine this with EEJ or $\mathbf{E} \times \mathbf{B}$ estimation mentioned above to address the connection between EEJ and ULF pulsation strength at low/mid-latitudes. We will perform both case studies, in particular during magnetically active periods as well as examining their connection statistically by binning the observations into LT, and geomagnetic activity bins. AMBER will also provide data to contribute to answering the role of ULF waves in the acceleration of $\mathrm{MeV}$ energy electrons as a function of magnetic activity. This could be done in collaboration with the radiation belt community using data from satellite(s) such as SAMPEX. 
We will also use the opportunity of the European magnetometer array extension to the dip-equator, through AMBER, to contribute to studies addressing another important space physics problem, i.e., the penetration of Pc5 ULF waves to low $L$-shell and its importance for the generation and acceleration of $\mathrm{MeV}$ energy "killer" electrons in the radiation belts. The picture is less clear at lower L-shells (e.g., Friedel et al. 2002), especially because classical ULF wave radial diffusion rates increase rapidly with $L$, typically $\sim L^{6}$ or faster (see, e.g., Loto'aniu et al. 2006). Since MeV energy electron flux enhancements are believed to have been responsible for a number of satellite failures and anomalies (see, e.g., Baker et al. 2004), this also has very important consequences for space weather. Understanding of the $\mathrm{MeV}$ energy electron flux enhancements mechanism is very important to the general knowledge of transport and heating processes in the magnetosphere. Therefore, AMBER will provide ULF wave power observations in the African sectors. At the same time, in collaboration with the radiation belt community, $\mathrm{MeV}$ relativistic electron flux propagating radially inwards from higher $L$-shell to lower $L$-shell will be obtained from satellite data. We will then be able to contribute to understanding the role of ULF waves in accelerating $\mathrm{MeV}$ relativistic electron especially at lower $L$-shells, this being of particulate importance for slot filling or inner zone enhancements (e.g., Baker et al. 2004). Loto'aniu et al. (2006) suggested that ULF waves might have contributed to slot and inner zone enhancements during the Halloween 2003 storms.

\section{Summary and Conclusion}

In collaboration with the radiation belt community, the NASA-IHY funded AMBER will also be used to understand the correlation between ULF wave and MeV energy electron fluxes. The outcome of this research will play a vital role for the efforts of complete and global understanding of the structure and dynamics of the equatorial ionosphere as well as for the effort of global modeling of the $\mathrm{N}_{\mathrm{e}}$ structure of the inner-magnetosphere. The US funded GPS receiver network in the region and the available LEO satellite GPS data will be used to image the regional plasma density structure of the inner-magnetosphere and to infer the $\mathrm{N}_{\mathrm{e}}$ structure and dynamics as a function of L-shell and altitude at low/midlatitudes in the African sectors using tomographic reconstruction technique. Therefore, the combination of AMBER magnetometer array and ground- and space-based GPS observation techniques will allow us to answer the following specific questions, of which most of them are unique in the African sectors according to satellite observation (Hei et al. 2005). These include: (1) why the ESF and thus equatorial bubbles are much deeper in the African region? (2) Why the bubbles in the African region are more active throughout the year compared to other longitudinal sectors? (3) Why the depleted plasma (or bubbles) penetrate to higher altitudes (up to $1,000 \mathrm{~km}$ sometimes) only in the African region? (4) What is the connection between bubble and anomaly, anomaly and $\mathbf{E} \times \mathbf{B}$, and ESF and $\mathbf{E} \times \mathbf{B}$ ? (5) How does the motion of the equatorial ionosphere in the African sector behave as a function of altitude, local time, latitude, season, and geomagnetic activity? (6) What is the connection between ULF pulsations at low L-shell and the strength of the EEJ and EA? By combining with $\mathrm{MeV}$ relativistic electron satellite data, in collaboration with radiation belt community, AMBER will play a vital role in answering another important science question. That is, are ULF waves the dominant mechanism for the generation and acceleration of MeV energy flux?

In addition to its contribution to the understanding of how human society, technological systems, and the habitability of the planets are affected by solar variability and planetary 
magnetic fields, which is one of the many NASA strategic science outcomes, AMBER array will play a vital role in fostering the international scientific cooperation in the study of helophysical phenomenon now and in the future. By filling the largest land-based gap in global magnetometer coverage in the African continent and connecting the European magnetometer array to the low- and mid-latitude region in the African local time sector, AMBER will allow the routine ground-based global observations that are important to coordinate with space-based missions such as THEMIS and CLUSTER. This will then provide a good opportunity to determine the response of terrestrial and planetary magnetospheres and atmospheres to external drivers. AMBER will also develop the basic science of heliophysics through cross-disciplinary studies of universal processes.

The AMBER project has direct impact on space science research and education both in developing nations and in the United States. It fosters international scientific cooperation by organizing research campaigns that establish research infrastructure or enhance existing research in the developing nations, which directly pertain to the IHY. In addition to facilitating the collection of world-class science data is also to ensure a legacy of IGY by providing a self-sustaining training and research infrastructure within African Universities during IHY period; and AMBER infrastructure will enable training to scientists from developing nations. At UCLA, AMBER project will create opportunities for undergraduate students to do research activities and that will provide them a high quality research experience. The experience will include expertise in data analysis, remote sensing, computer modeling, data assimilation, and grid-based computing and data retrieval.

Acknowledgments This research work has been financially supported by National Science Foundation (NSF) grants ATM-0524711 and ATM-0348398, National Aeronautics and Space Administration (NASA) grant NNX07AM22G.

Open Access This article is distributed under the terms of the Creative Commons Attribution Noncommercial License which permits any noncommercial use, distribution, and reproduction in any medium, provided the original author(s) and source are credited.

\section{References}

C. Amory-Mazaudier et al., J. Atmos. Solar-Terr. Phys. 67, 269 (2005)

D.N. Anderson, Planet. Space Sci. 21, 3 (1973)

D. Anderson, A. Anghel, K. Yumoto, M. Ishitsuka, E. Kudeki, Geophys. Res. Lett. 29, 12 (2000)

D.N. Baker, S.G. Kanekal, X. Li, S.P. Monk, J. Goldstein, J.L. Burch, Nature 432, 378 (2004)

N. Balan, G.J. Bailey, J. Geophys. Res. 100, A11 (1995)

D. Berube, M.B. Moldwin, J. Weygand, J. Geophys. Res. 108, A9 (2003)

B.G. Fejer, L. Scherliess, E.R. de Paula, J. Geophys. Res. 104, A9 (1999)

R.H.W. Friedel, G.D. Reeves, T. Obara, J. Atmos. Solar-Terr. Phys. 64, 265 (2002)

M.A. Hei, R.A. Heelis, J.P. McClure, J. Geophys. Res. 110, A12315 (2005)

S. Heise, N. Jakowski, A. Wehrenpfennig, Ch. Reigber, H. Lühr, Geophys. Res. Lett. 29, 14 (2002)

R.B. Horne et al., Nature 437, 227 (2005)

J.A. Klobuchar, J.M. Goodman, H. Soicher, Radio Sci. 26, 4 (1991)

T.M. Loto'aniu, I.R. Mann, L.G. Ozeke, A.A. Chan, Z.C. Dent, D.K. Milling, J. Geophys. Res. 111, A04218 (2006)

I.R. Mann, T.P. O’Brien, D.K. Milling, J. Atmos. Solar-Terr. Phys. 66, 187 (2004)

A.J. Mannucci et al., Geophys. Res. Lett. 32, L12S02 (2005)

M. Mendillo, J. Meriwether, M. Biondi, J. Geophys. Res. 106, A3 (2001)

B.M. Pathan, N.G. Kleimenova, O.V. Kozyreva, D.R.K. Rao, R.L. Asinkar, Earth Plan. Space 51, 947 (1999)

P. Ritter, H. Lühr, A. Viljanen, O. Amm, A. Pulkkinen, I. Sillanpää, Ann. Geophys. 22, 417 (2004)

G. Rostoker et al., Space Sci. Rev. 71, 743 (1995) 
E. Sardón, A. Rius, N. Zarraoa, Radio Sci. 29, 577 (1994)

J.D. Tarpley, Planet. Space Sci. 18, 1091 (1970)

N.A. Tsyganenko, J. Geophys. Res 107, A8 (2002)

E. Yizengaw, E.A. Essex, R. Birsa, Ann. Geophys. 22, 8 (2004)

E. Yizengaw, M.B. Moldwin, Geophys. Res. Lett. 32, L09105 (2005)

E. Yizengaw, P.L. Dyson, E.A. Essex, M.B. Moldwin, Ann. Geophys. 23, 3 (2005a)

E. Yizengaw, M.B. Moldwin, P.L. Dyson, T.J. Immel, J. Geophys. Res. 110, A09S30 (2005b)

E. Yizengaw, M.B. Moldwin, P.L. Dyson, B.J. Fraser, S.K. Morley, Geophys. Res. Lett. 33, L20102 (2006)

E. Yizengaw, M.B. Moldwin, P.L. Dyson, E.A. Essex, J. Atmos. Solar-Terr. Phys. 69, 314 (2007)

K. Yumoto, 36th Committee on Space Research (COSPAR) Scientific Assembly (2006)

E. Zesta, A. Boudouridis,D. Berube, M. Moldwin, Eos Trans. AGU, 85(47), Fall Meeting, Suppl., Abstract SM21A-0455 (2004) 